\title{
Biblioteca pública y las necesidades de información de la comunidad
}

\author{
Emir José Suaiden*
}

\begin{abstract}
RESUMEN
En América Latina la mayoría de las bibliotecas públicas funcionan hasta hoy como bibliotecas escolares y con pocas excepciones no se puede afirmar que la biblioteca pública tenga realmente un trabajo eficaz en pro de la comunidad. La gran prueba es que la comunidad no ve, aún hoy, a la biblioteca como una institución indispensable en los planes de desarrollo de una nación. Para cambiar esta situación los profesionales de la información necesitan utilizar metodología adecuada para diagnosticar las necesidades de información y trabajar cada vez más con la información a la comunidad.
\end{abstract}

\section{ABSTRACT}

In Latin America most public libraries operate as school libraries and it can be said that the public library has not had a great impact on the community, except in few cases. This is confirmed by the attitude of the community that still do not see the library as an essential institution for development plans in a country. To change this situation the information professionals need to use an adequate methodology to diagnose the needs for information and work more and more with this information for the community.

$\mathrm{E}_{\mathrm{b}}^{\mathrm{n}}$ América Latina y el Caribe, los gobiernos se enfrentan con una serie de problemas, como la desnutrición, la mortalidad infantil, la deuda externa, el analfabetismo, etc. Todos estos problemas dificultan una prioridad mayor para los sectores de la educación y de la cultura.

En muchos países la biblioteca pública se desarrolla gracias a la determinación y creatividad de los bibliotecarios que creen en su institución como agente de transformación de la sociedad.

Podríamos afirmar que la biblioteca, así como los esfuerzos de los bibliotecarios, hasta el momento, se han visto envueltos en numerosos problemas: económicos, de recursos humanos no siempre adecuados; falta de interés por parte de las autoridades; y problemas relativos a la planificación de actividades/servicios de divulgación, entre otros. Todo lo anterior ha conducido a que la biblioteca haya contribuido de forma escasa al desarrollo cultural y educativo de la comunidad a la cual sirve.

La biblioteca pública al vincularse y dar prioridad a una fuente de información/conocimiento (el libro) y a un solo tipo de servicio (la lectura), que depende de la fuente antes indicada, dejó de atender a importantes sectores de la comunidad, los cuales precisaban una información oral o de naturaleza ciudadana que en la mayoría de los casos no se encuentra impresa ni registrada en libros. Aquí podríamos incluir, por ejemplo, las poblaciones marginadas (particularmente los grupos de analfabetas), que en Brasil llegan a representar una parte significativa de la población, y para los que la información bibliográfica no es de tanta importancia.

Por otra parte, al localizarse en el centro urbano y con falta de servicios de extensión que atiendan a las poblaciones residentes en el periferia, la biblioteca dejó de atender también a ciertas poblaciones suburbanas y rurales que necesitaban los servicios bibliotecarios con la misma perentoriedad que las que residían en el centro, pues también para aquellas, el libro acceso a la información, es un requisito previo a la formación de comunidades conscientes, integradas a la cultura de su nación, adecuadas a su tiempo y capacitadas para encontrar un equilibrio a todas las ideologías y tendencias que actúan en la sociedad contemporánea.

La falta de divulgación y el aprovechamiento de los medios de comunicación de masas para llevar a cabo esta actividad, aunada a la falta de mecanismos adecuados para promover los servicios, hizo que el gran público no se interesara por el potencial de recursos y servicios que la biblioteca pública ofrecía. Pero además, la falta de colaboración entre las bibliotecas públicas ocasionó costos económicos elevados para la correcta atención de las necesidades y la actualización de los fondos bibliográficos, a lo que se

* Doctor en ciencias de la Información 
unió la correcta distribución de infrautilización de los existentes.

Por otra parte, no debemos olvidar que el profesional bibliotecario recibe muchas veces una formación inadecuada en las escuelas de bibliotecología. No son instruidos para diagnosticar las necesidades de información de la comunidad, trabajan solamente con información impresa o de carácter bibliográfico y salen de las escuelas con la convicción de que existe de forma generalizada un hábito de lectura, surgiendo después una decepción cuando han de enfrentarse al mercado de trabajo y a la realidad de la comunidad.

Todos estos problemas convirtieron la imagen de la biblioteca pública en la de una institución pasiva ante los problemas de la comunidad y, también pasivamente, la biblioteca pública aceptó asistir y dar prioridad en sus servicios a un único sector de la comunidad, que es de estudiantes, sin buscar mecanismos adecuados para atender a otros sectores como el de amas de casa, jubilados, desempleados, emigrantes, trabajadores, etc. La mayoría de los estudiantes utilizan la biblioteca pública para realizar las tareas escolares, cuando el objetivo de la biblioteca es el de ser una institución de transformaciones sociales, agente principal de una política de formación de hábitos de lecturas y uso de la información.

Para cambiar la situación actual, la biblioteca pública necesita urgentemente utilizar las metodologías de estudio de usuarios y de estudio de no usuarios (anexo).

Con relación a los estudios de usuarios es necesario destacar dos trabajos de gran importancia, los estudios de D. Elia ${ }^{3}$ y Madden. ${ }^{9}$

Según investigaciones recientes son los estudiantes los grandes usuarios de las bibliotecas públicas. Pero del gran porcentaje que representan los estudiantes entre los lectores de las bibliotecas públicas, se deducen también dos importantes conclusiones:

a. La parte de la comunidad que queda, excluyendo a los estudiantes, incluye a los trabajadores, obreros de la construcción, los industriales, amas de casa, comerciantes, etc. Todos ellos tienen una participación poco signifi- cativa en relación con la frecuencia con que asisten a la biblioteca pública o usan los recursos que ésta les ofrece.

b. La participación efectiva del estudiante es muy importante en la biblioteca pública y esto significa, cuando menos, que los futuros administradores por haber frecuentado la biblioteca pública sabrán valorar mejor la función de esta institución en el plan de desarrollo nacional, pues según Banberger" "el derecho a leer significa igualmente el derecho a desarrollar las capacidades intelectuales y espirituales de la persona, el derecho de aprender y progresar".

No obstante, si analizamos el porcentaje de estudiantes que no frecuentan la biblioteca pública, llegaríamos ala conclusión de que se trata de un porcentaje muy elevado. En realidad, pocos son los países en los que el sistema de enseñanza favorece a la biblioteca, ya que los propios profesores no fueron educados para utilizarla.

Con creatividad el bibliotecario puede aprovechar la permanencia del estudiante en el recinto de la biblioteca para diversificar sus intereses de lecturas y así contribuir eficazmente para la formación de un público lector.

Con relación a los no usuarios, el porcentaje de ellos es bastante superior al de usuarios. Generalmente, los no usuarios viven en comunidades alejadas de los centros urbanos y se enfrentan además con la falta de bibliotecas públicas, con los problemas económicos, culturales y educativos. Son las llamadas poblaciones marginadas. En Brasil es común la formación de casetas (chabolas), en los que no hay condiciones mínimas de higiene y servicios públicos como la luz, agua, teléfono, etc. La gran mayoría de esas personas, en Brasil, son analfabetas, y no tienen empleo.

La organización y eficacia de los servicios bibliotecarios o de información es de fundamental importancia para la creación de los hábitos de necesidad informativa. Nice Menezes de Figuereido ${ }^{6}$ cita las barreras y factores que causan la infrautilización de la información: a. Acceso a la información:

- falta de controles bibliográficos a nivel nacional;

- barreras lingüisticas;

- falta de servicios de referencia;

- falta de recursos para la adquisición de fuentes/bases de datos;

b. Disponibilidad de documentos:

- falta de un catálogo colectivo nacional;

- falta de un depósito y reposición de documentos;

- restricciones políticas, de seguridad nacional de copyright.

- Absorción y uso eficaz de la información:

- falta de entrenamiento del usuario;

- falta de promoción del uso de la información;

- prestaciones deficientes de los servicios/sistemas de información.

Para la promoción del uso de la información, esta misma autora propone que se realice:

a. Un estudio de usos/usuarios, definiendo hábitos, motivaciones, necesidades, percepciones y actitudes con relación a sus necesidades y a los servicios/productos ya existentes.

b. La evaluación de los recursos informativos existentes para atender las demandas de información.

c. Un análisis del espacio físico y de la disposición general de la colección/servicio, para acondicionarlo a las necesidades y prioridades que requiera la difusión de la información.

d. Actualización permanente del personal, entrenándolos en la prestación de servicios de referencia/información y preparación de los instrumentos de difusión de la información; 
e. Educar al usuario para un uso eficiente de los recursos informativos disponibles en su área de actuación.

f. Uso del marketing y promoción de los servicios/productos a través de guías, folletos divulgativos, contactos, comunicación, campañas, etc.

Las necesidades de información de los no-usuarios son muy distintas y comprenden lo que podríamos denominar como información utilitaria, destinada a resolver los problemas cotidianos. Ana María Polke ${ }^{5}$ bibliotecaria dedicada en gran parte al estudio de esta información utilitaria, la estructura en seis aspectos esenciales:

1. Salud: problemas de asistencia médica y hospitalaria; cómo, dónde y a quién recurrir para la solución de los problemas referidos a la salud; planificación familiar, prevención de dolencias, vacunación.

2. Empleo: problemas para obtener trabajo, estabilidad o fluctuación, agencias, conciliación de las actividades de la casa con las tareas domésticas.

3. Legislación: problemas de obtención de documentos, conocimiento de derechos y deberes legales, asistencia jurídica, existencia de asociaciones de vecinos, jubilación y obtención de beneficios.

4. Educación: problemas de plazas escolares, abandono de la escuela, reprobación, alfabetización de adultos, formación profesional, obtención de becas, orientación sexual, educación para adultos (escuelas de padres, trabajos manuales, artesanales...);

5. Ocio: problemas relacionados con el tiempo libre, cuáles son los tipos preferidos de distracción, obstáculos al ocio, papel de la televisión y la radio; lectura para el ocio (qué, cómo y para qué leer);

6. Vivienda: problemas de posesión de la tierra, alquiler, acondicionamiento de vivienda, invasión de terrenos, servicios de agua, desagüe y luz, condiciones de residencia, vecindad...
La referida bibliotecaria llegó a esas conclusiones basándose en un estudio realizado en el barrio de Pompeya, en Belo Horizonte (Brasil), teniendo como objetivo de análisis saber cuáles son las informaciones más necesarias para la vida cotidiana; y cuáles son las dificultades con que se enfrentaban en su búsqueda. Las respuestas muestran que la información más usada por esta población es la oral, obtenida generalmente de vecinos, amigos, y parientes. Mientras que en los niveles socio-económicamente superiores la información registrada o impresa es utilizada para complementar la información oral; en los niveles más bajos eso no se da, debido al analfabetismo, la falta de hábito de lectura y el bajo poder adquisitivo.

La obtención de la información es tanto más difícil y penosa cuanto más bajo es el nivel socio-económico del individuo que la busca.

La demanda de información en el área de empleo ha revelado ser la más apremiante y donde la comunidad se encuentra con mayores obstáculos. Existen instituciones que prestan asistencia, ofertas de empleo), pero, en la opinión de los que la utilizan, no atienden sus necesidades. El gran "no público" de la Biblioteca de Pompeya indica al bibliotecario las posibilidades de atención que se requieren para provisión de información utilitaria.

El desempleo hace que todos los años millares de emigrantes del norte y noroeste brasileño vayan a São Paulo y Brasilia en busca de mejores condiciones de vida. LA gran mayoría son obreros sin especialización y analfabetos. En el "Estudio de las necesidades de información de los emigrantes del noroeste brasileño que fueron a São Paulo y Brasilia", realizado por Angele R. F. A. Dos Santos y otros, ${ }^{6}$ el objetivo era diagnosticar "si la falta de información pudo ser uno de los factores que motivaron a esta gente a no permanecer en tales ciudades".

Los objetivos específicos del mencionado estudio eran averiguar si existía una necesidad de información por parte de los emigrantes, determinar cuál era la que requerían para asentarse en una región que no era la suya de origen y diagnosticar los factores que determinaron el

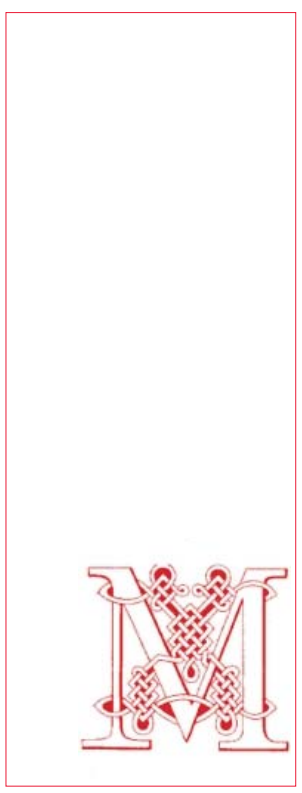

"La obtención de la información es tanto más difícil y penosa cuanto más bajo es el nivel socio-económico del individuo que la busca" 
grado de información de los emigrantes. Además, el estudio se proponía evaluar las condiciones que concurrieron para su residencia en tales ciudades; así como también caracterizar al grupo de emigrantes que llegó a Brasilia y São Paulo, los cuales finalmente no se instalaron; lo mismo que detectar las diferentes necesidades de información entre los que acudieron a una y otra ciudad y definir los servicios básicos de información necesaria para su adaptación social en estas localidades. Por último se intentaba comprobar si los servicios de asistencia al grupo de emigrantes fueron satisfactorios, proponiendo la participación de la biblioteca en un servicio no convencional de información utilitaria compatible con el tipo de necesidad de información de esos emigrantes.

El estudio presentaba también una revisión de la literatura, con la definición y caracterización que del emigrante se hacía, así como las causas y motivos de las emigraciones; la selectividad migratoria; la región noroeste del Brasil y el emigrante propio de esa zona; la afluencia del mismo hacia São Paulo y Brasilia; y la adaptación del emigrante en un medio social urbano; además de la política de emigración; el papel social de la biblioteca y la información comunitaria utilitaria.

El siguiente paso consistió en presentar la metodología, con los parámetros utilizados para medir la falta de información en los emigrantes del noroeste, la limitación de estudios y el proceso de recopilación de datos, que en este caso se realizó con una entrevista estructurada.

El análisis continuaba con una interpretación de los datos, ofreciendo en el último capítulo algunas conclusiones y sugerencias. La metodología presentada en el referido trabajo sirve de modelo para diagnosticar necesidades de información en la comunidad. En realidad para los emigrantes que llegaban a São Paulo era poco importante que la Biblioteca Municipal "Mario de Andrade" fuera la principal de América Latina y tuviera dos millones de libros en su fondo. Los emigrantes no necesitaban la información bibliográfica, pero sí la referida a la educación de su hijos, salud, vivienda y principalmente cuestiones de empleo.
Por otra parte, debemos contemplar también el hecho de que el número de analfabetos en Brasil es muy grande, lo cual conduce a la falta de interés que este tipo de personas tienen por la información bibliográfica y de ahí que sean no usuarias de las bibliotecas públicas.

Cuando se habla de educación y bibliotecas destinadas a personas marginadas, no podemos olvidar a Paulo Freire.

Paulo Freire ${ }^{7}$ se basa en la afirmación ontológica de que todos los hombres son iguales ante la naturaleza y el conocimiento, por lo que el tratamiento que debe dispensarse al adulto en su práctica educativa se centra en este mismo aspecto. No hay hombre ignorante en su totalidad, en quien falta un conocimiento sistematizado, pues "el hombre, por ser inacabado, incompleto, no sabe de manera absoluta", y como un ser inacabado está en constante búsqueda, ya que nadie ignora todo, nadie sabe todo". 8 La preocupación de Freire se cierne en la participación y emancipación de la persona. Destaca fuertemente una valoración positiva del hombre y una fe en su libertad y en su poder de creación y crítica.

Freire señala también la necesidad del hombre de tener conciencia de su lugar en la sociedad. Para sustituir la realidad de la escuela, propone el ámbito de la lectura, que es un contexto más flexible y dinámico. En lugar del profesor, aparece el moderador de debates y su papel es el de crear los estímulos necesarios para el acto de conocimiento, donde, justamente con el educando, se procura reflejar la situación real desde una postura crítica. Las enseñanzas de Paulo Freire son de gran importancia para la biblioteca pública por dirigirse a las clases marginadas de la sociedad y especialmente de los analfabetos.

Como ya dijimos, el porcentaje de los no usuarios es altamente superior al de los usuarios en las bibliotecas públicas. Para atraer a esa nueva e importante clientela es necesaria una nueva postura por parte de los profesionales de la información, pues, en muchas regiones, el propio personal de la biblioteca pública ha contribuido a que las personas marginadas des- de el punto de vista educativo, económico y social, no utilicen la biblioteca. Al ser construida en el centro de la ciudad, la biblioteca facilita el acceso de los habitantes de la zona urbana, pero hasta hoy no ha conseguido ampliar los servicios a la periferia, a la zona rural, hospitales y cárceles, etc. Muchas bibliotecas públicas han pasado a reservar un espacio físico para las academias de letras, los intelectuales..., pero jamás se han preocupado de promover reuniones con los trabajadores de la construcción civil o con los habitantes de las casetas para discutir problemas relacionados con la vivienda o cono la dignidad humana. Debido a este tipo de comportamiento, las poblaciones se han ido desmotivando paulatinamente para frecuentar la biblioteca. Ahora bien, es verdad que el primer contacto del individuo con la biblioteca debe ser hecho por medio de la escuela en los primeros años de la enseñanza y, muchas veces, la falta de bibliotecario no ha hecho posible la concreción de esa iniciación en la biblioteca. Con pocas excepciones, no ha habido promoción o divulgación de los servicios bibliotecarios y el marketing tampoco ha llegado a ser utilizado por la biblioteca pública, por lo que la mayoría de la población desconoce las actividades bibliotecarias.

Ha llegado la hora de que los bibliotecarios se unan a otros profesionales, como sociólogos y psicólogos, para investigar las barreras que separan a los no usuarios de la biblioteca pública. En realidad está en primer lugar el problema económico, pues la población menos favorecida tiene obligatoriamente que tener más de un empleo, o dedicarse al comercio ambulante para sobrevivir. Con ello no queda tiempo para satisfacer las necesidades intelectuales. Debe considerarse también la inhibición natural de esas poblaciones cuando entran en una biblioteca pública para solicitar informaciones.

Por otra parte, la falta de tiempo y escasez de información necesaria son seguramente las disculpas más comunes de las personas que no utilizan la biblioteca pública. Pero en este círculo vicioso la biblioteca tiene también culpa, pues en la mayoría de los pueblos no prolonga el horario de atención al público usuario. Si la comunidad no encuentra realmente 
la información que requiere, el problema es muy grave, pues no es correcto ya denominar a esta institución "biblioteca pública". Si el principal objetivo de esta institución es el de mantener un fondo suficiente y adecuado, y brindar las informaciones que la comunidad necesita tenemos que reconocer, sin embargo, que en algunos casos la biblioteca posee la información, pero, por falta de divulgación, las personas no adquieren conocimiento del potencial de sus servicios.

Este distanciamiento de la biblioteca con la comunidad hace que las personas utilicen otras fuentes para satisfacer sus necesidades de información acudiendo frecuentemente a intelectuales, profesores y directores de escuela, con lo que la biblioteca pierde poco a poco su objetivo y última meta de ser el gran centro de difusión de la información. Ocurre actualmente que falta una motivación para que el gran público frecuente la biblioteca, y el bibliotecario, quizá por falta de una información más adecuada, por carecer de creatividad y sentido común no consigue promover ni despertar esa motivación.

La implantación del servicio de información a la comunidad por medio de las bibliotecas públicas, tomando como base el diagnóstico de las necesidades de información de la comunidad y con una amplia interacción entre las partes (biblioteca y comunidad) puede ser de gran importancia para el desarrollo de todos los sectores de la comunidad. Permitirá a la biblioteca pública una nueva dimensión de su servicio.

En la medida en que la interacción con la comunidad sea eficaz, la imagen de la biblioteca mejorará en todos los aspectos, incluso en la relación de la misma con las autoridades, posibilitando asimismo un incremento de los recursos asignados, además de contar también con los de la comunidad. Sólo así, la biblioteca pública podrá ser el gran centro de difusión de la información y pasará a ser realmente una institución de, y, para la comunidad. Esto presupone a su vez un carácter democrático en todos los niveles que constituyen la biblioteca.

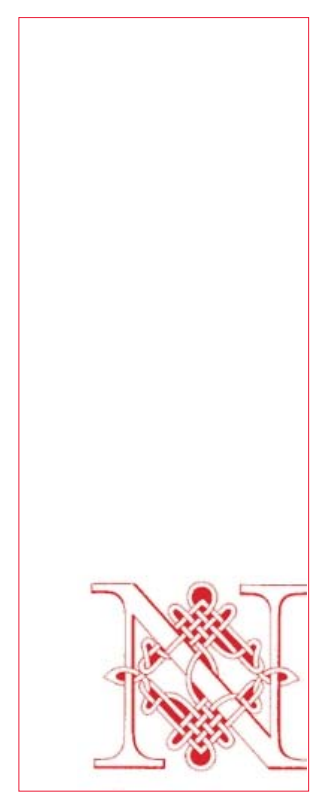

\section{"Si la comunidad no encuentra realmente la información que requiere, el problema es muy grave, pues no es correcto ya denominar a esta institución biblioteca pública”}

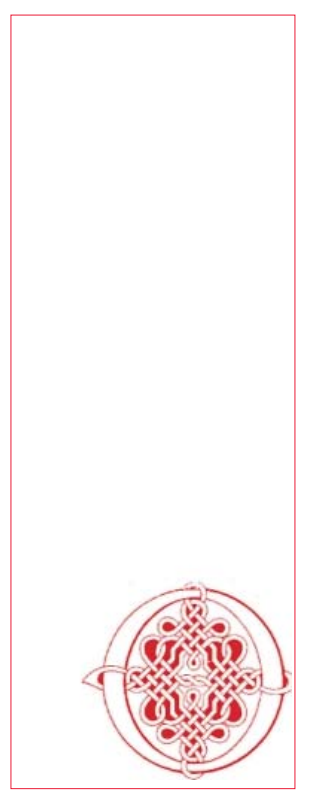

Por otra parte, la comunidad deberá estar presente en todas las decisiones que cada nivel adopte, y los fondos de la biblioteca, habrán de reflejar las necesidades de la comunidad. El análisis de la comunidad tiene como fin el conocimiento del entorno de la biblioteca y la identificación de las necesidades de los ciudadanos en relación con la oferta bibliotecaria. Este análisis, realizado sistemáticamente, sirve de ayuda para argumentar y, sobre todo, para planificar, además facilita la fijación de objetivos y el establecimiento de prioridades, ofrece la base para una oferta dirigida por la demanda, es útil para el marketing y las relaciones públicas, así como para decisiones sobre ubicación y distribución del espacio en las bibliotecas, niveles de personal, horarios al público y organización.

Con estas características, la biblioteca pública se convertirá también en una institución pública realmente, pues pasará a difundir informaciones a todos los estratos de la población, lo cual es de suma importancia si consideramos que la mejora en la salida de vida puede alcanzarse gracias a la información actualizada, lo cual puede coadyuvar a que las personas consigan puestos escolares, becas de estudios, documentos, y tantos otros derechos que la comunidad les confiere.

La interacción y vinculación con la comunidad repercutirá en que la biblioteca pública pase a ser una institución indispensable en los planes de desarrollo social, cultural y educativo de un país. Su característica de neutralidad respecto a la situación política conducirá asimismo a una formación de las personas de forma más integrada, capaces de valorar las riquezas culturales del propio país.

Por otra parte, en nuestra opinión, sólo una integración efectiva de la biblioteca pública en el sistema de enseñanza podrá favorecer el cambio de la situación actual. La referida integración permitirá que el profesor y el bibliotecario trabajen juntos con el interés de formar personas en quienes tanto la investigación bibliográfica como el espíritu crítico, sean ambos, elementos fundamentales en la difusión del hábito de lectura y en la utilización de la información. 
Pero si la biblioteca persigue ser pública ha de ser también actual, y su integración en la comunidad ha de interesarse no sólo por el diagnóstico de las necesidades de información que ésta posea, sino también por el uso del tiempo de ocio por parte de sus miembros o incluso por necesidades de información sobre temas que no se vinculan directamente con los servicios bibliotecarios. La realización periódica de charlas, conferencias, debates, etc., por parte de la biblioteca pública es también una manera de servir a la comunidad.

También es importante que la biblioteca se relacione con los medios de comunicación de masas, y esto no sólo para divulgar las actividades, sino también para promover al máximo un trabajo motivado por las necesidades de la comunidad.

A su vez, la nueva postura de la biblioteca, que se integra en la comunidad, deberá posibilitar que, a corto plazo, la currí- cula académica de las escuelas de bibliotecología incluya asignaturas con vistas a promover cada vez más esa integración mediante un estudio de la metodología apropiada.

Por último cabe señalar dos aspectos más. El primero se relaciona con una frase que se utiliza con bastante frecuencia: "democracia de la información". Esto presupone que todas las personas tienen el derecho de recibir la información que precisen, independientemente de su lugar de residencia y de otros factores. Por ello es fundamental en el servicio bibliotecario que, a través de servicios de extensión, se facilite el acceso a la información a personas que viven en zonas periféricas y rurales, En segundo lugar, es importante también, y en gran medida tiene que ver con el aspecto anterior, que la recopilación, almacenamiento y difusión de la información posean un máximo de eficacia que contribuya a que la in- formación esté disponible en el menor tiempo posible.

Como resumen de todo lo dicho en páginas anteriores cabría finalmente indicar que la implantación de los servicios de información a la comunidad en las bibliotecas públicas sería de fundamental importancia, al contribuir a que se eliminen las barreras que separan a ciertos grupos de la información, lo cual puede conducir a la ignorancia por parte del individuo, de sus derechos y deberes ante la sociedad, dificultando también una mejora global de la calidad de vida. Esperamos con este trabajo haber contribuido, aunque sea en forma básica a la implantación de ese servicio que, en nuestra opinión, tantos bienes puede acarrear a la vida social y cultural de un país.

\section{NOTAS BIBLIOGRÁFICAS}

1. BANERGER, R. Como incentivar o hábito de leitura. Sao Paulo, Cultrix, 1977. p. 25.

2. BARBOZA, J. P. A biblioteca pública como alternativa de educação não formal para adultos analfabetos. João Pessoa, UFPB, 1984 (Dissertação de Mestrado, UFPB).

3. D. ELIA G.P.M. "User satisfaction with library service an easier of public library service?" Library Quaterly. 54 (2), 1983: 109-133.

4. DELON DE QUINTERO, Ana. Orientaciones para la creación, organización y funcionamiento de servicios de información a la comunidad en América Latina y el Caribe. Caracas, UNESCO, 1987.

5. FALCON, Aevys. Servicio Nacional de Información a la Comunidad: manual de procedimientos. Caracas, IABN, 1984.

6. FIGUEIREDO, N. M. "Da necesidad de promover o uso da informação". Ciencia de Informação. 16 (1)], 1987: 75-80.

7. FREIRE, P. Educação como prática de liberdade. Rio de Janeiro, Paz e Terra, 1981.

8. FREIRE, P. Alfabetização de adultos e bibliotecas populares. São Paulo, Autores Asociados, 1982. 
9. MADDEN, M. "Library user/nonuser lifestyles". American Library. 10(2), 1979: 78-81.

10. POLKE, A. M. A. "Biblioteca, comunidade e informação utilitaria no Bairro de Pompéia, em Belo Horizonte". In: $C B B D, 11$. Anais: Joãp Pessoa, APBP. 1982: 131-159.

11. SANTOS. Angela et al. "Estudo das necesidades de informação dos migrantes nordestitos que chegam a São Paulo e Brasilia". In Revista de Biblioteconomía de Brasilia, 12 (2) 1984: 229-259.

12. SUAIDEN, Emir J. Información a la comunidad: modelo de planificación e implantación del servicio en bibliotecas públicas de España y Brasil. Madrid, Universidad Complutense de Madrid, 1989: 314 p. 


\section{ANEXO}

\section{CUESTIONARIO}

\section{PRESENTACIÓN}

Este cuestionario pretende detectar, a través de la recolección de datos para un posterior muestreo, las necesidades de información de la comunidad. Está dividido en cuatro partes:
1. Parte: datos de identificación
$2^{a}$. Parte: datos sobre los usuarios de las bibliotecas públicas.
3a. Parte: datos sobre los no-usuarios de las bibliotecas públicas.
4 . Parte: datos sobre información a la comunidad

\section{$1^{\text {a }}$. Parte. Datos de identificación}

\subsection{Profesión}

$\begin{array}{ll}\text { - Estudiante: } & \text { EGB } \\ & \text { BUP } \\ & \text { COU } \\ & \text { Universitario } \\ & \text { Graduado }\end{array}$

- Trabajador: Sí

No

- Otros: $\quad$ Citar

1.2. Edad

- 06 a 13 años

- 14 a 17 años

- 18 a 25 años

- 26 a 40 años

- Más de 40 años

1.3. Nivel socio-económico

$$
\begin{aligned}
& \text { - Alto } \\
& \text { - Medio } \\
& \text { - Bajo }
\end{aligned}
$$

\section{$2^{\mathrm{a}}$. Parte. Datos sobre los usuarios de la Biblioteca Pública}

2.1. Frecuenta la biblioteca pública

- Diariamente

- Semanalmente

- Mensualmente

- Algunas veces

2.2 ¿Qué tipo de servicio utiliza en la biblioteca pública?

- Préstamo a domicilio

- Consultas

- Bibliografías

- Fotocopias

- Audiovisual

- Otros:

Citar:

2.3. ¿Qué tipo de material utiliza en la biblioteca pública?

- Libros didácticos

- Libros de texto

- Libros de ficción

- Folletos

- Revistas

- Diarios

- Audiovisuales

- Referencia (diccionario, enciclopedias, atlas,

mapas, bibliografías, guías, normas, etc.)

- Otros: Citar:

2.4. ¿Satisface sus necesidades de información la biblioteca pública?

$$
\begin{aligned}
& \text { - Siempre } \\
& \text { - Frecuentemente } \\
& \text { - Algunas veces } \\
& \text { - Nunca }
\end{aligned}
$$


2.5. Además de biblioteca publica, ¿utiliza otras instituciones o acude a personas que respondan a las necesidades de información?
- Biblioteca escolar
- Biblioteca especializada
- Biblioteca nacional
- Profesor
- Amigos
- Familiares
- Otros: Citar:

2.6. ¿Participa en otras actividades de la biblioteca pública?

- Seminarios
- Debates
- Hora del cuento
- Películas
- Reuniones
- Exposiciones
- Cursos
- Otros: Citar:

2.7 ¿Cuáles son las deficiencias que la biblioteca pública presenta?

- Distanciada de casa

- Falta de espacio

- Mobiliario inadecuado

- Fondos no actualizados

- Mala iluminación

- Desinterés del bibliotecario por atender a los lectores

- Burocracia que dificulta la atención a los usuarios

- Instalaciones inadecuadas

- Falta de personal capacitado

- Griterío, ruido

- Horario inadecuado

- Sistema de entrada

- Catálogos complicados

- Mala señalización
- Préstamo a domicilio inadecuado con relación al número de volúmenes

- Préstamo a domicilio inadecuado con relación al período de préstamo

- Pago de cuotas para asociarse

- Ausencia de catálogos adecuados

- Otros: Citar:

\section{Parte. Datos sobre los no usuarios de la biblioteca pública}

3.1 Indique las razones por las que no frecuenta la biblioteca pública

- Falta de motivación

- Distanciada de casa

- La biblioteca pública no tiene la información que necesita

- Burocracia para tender al usuario

- Horario inadecuado

- Falta de tiempo

- Falta de divulgación de los servicios de la biblioteca pública

- Falta de medios económicos para asociarse a la biblioteca pública

- Falta de medios económicos para pagar el transporte

- Otros: Citar:

3.2. ¿Cómo obtiene la información que necesita?

- Amigos

- Familiares

- Profesores

- Biblioteca escolar

- Biblioteca especializada

- Libreros

- Otros: Citar:

3.3. ¿De qué forma obtiene la información?

- Conversando (información oral)

- Consultando libros (información)

-Televisión

- Emisoras de radio

- Otros:

Citar: 


\section{$4^{\text {a }}$. Parte. Datos sobre información a la comunidad}

Información a la comunidad, también llamada información utilitaria o información ciudadana, es un servicio que puede ser prestado por la biblioteca pública objetivando la divulgación de informaciones para que las personas puedan resolver sus problemas diarios, como: obtener documentos, conseguir empleos, saber la programación de cine, teatro, televisión u otras actividades culturales, estar informado de las actividades del gobierno, etc.

1.4 Cite, de las informaciones abajo señaladas, las que considera importantes para que las difunda la biblioteca pública.

- Urgencia (bomberos, policía, hospitales, farmacias de guardia, etc.)

- Asistencia sanitaria (hospitales, clínicas, centros de salud, puestos de vacunación, etc.)

- Asistencia social (a menores, anciano, mujeres, etc.)

- Asistencia legal (juzgados, tribunales, prisiones, servicio de asistencia jurídica gratuita, etc.)

- Empleo (obtención de empleo, oficinas de empleo, oposiciones, estabilidad y fluctuación en el empleo, derechos y obligaciones del trabajador, etc.)
- Servicios Públicos (agua, luz, gas, teléfono, transporte, etc.). Actividades culturales (museos, bibliotecas, galerías de arte, centros de congresos, de exposiciones, monumentos históricos, conciertos, teatros, cines, televisión, etc.)

- Organismos oficiales (Presidencia de Gobiernos, Parlamento, Ministerios, Administración Estatal, Autónomos, Local, etc.) Organizaciones (deportivas, culturales, sindicales, religiosas, etc.)

- Calendario de acontecimientos (espectáculos, congresos, juegos, etc.)

- Personalidades nacionales (pintores, escritores, escultores, músicos, políticos, etc.)

- Información educativa (dónde estudiar, becas, instituciones educativas y científicas, etc.)

- Trámites y procedimientos (requisitos para obtener el carnet de identidad, pasaporte, cartilla militar, registro civil de nacimiento, empadronamiento, etc.)

- Otros: Citar: 